\title{
The human immunodeficiency virus preventive vaccine research at the French National Agency for acquired immunodeficiency syndrome research
}

\author{
Elizabeth Fischer ${ }^{+}$, Véronique Rieux, Jean-Gérard Guillet, Michel Kazatchkine
}

Agence Nationale de Recherches sur le SIDA, 101 rue de Tolbiac, 75013 Paris, France

The human immunodeficiency virus/acquired immunodeficiency syndrome (HIV/AIDS) epidemic is of unprecedented gravity and is spreading rapidly, notably in the most disadvantaged regions of the world. The search for a preventive vaccine is thus an absolute priority. For over 10 years the French National Agency for AIDS research (ANRS) has been committed to an original program combining basic science and clinical research. The HIV preventive vaccine research program run by the ANRS covers upstream research for the definition of immunogens, animal models, and clinical research to evaluate candidate vaccines. Most researchers in 2004 believe that it should be possible to obtain partial vaccine protection through the induction of a strong and multiepitopic cellular response. Since 1992, the ANRS has set up 15 phases I and II clinical trials in order to evaluate the safety and the capacity of the candidate vaccines for inducing cellular immune responses. The tested candidate vaccines were increasingly complex recombinant canarypox viruses (Alvac) containing sequences coding for certain viral proteins, utilized alone or combined with other immunogens (whole or truncated envelope proteins). ANRS has also been developing an original strategy based on the utilization of lipopeptides. These comprise synthetic fragments of viral proteins associated with lipids that facilitate the induction of a cellular immune response. These approaches promptly allowed the assessment of a prime-boost strategy combining a viral vector and lipopeptides.

Key words: human immunodeficiency virus - vaccine trials - lipopeptides - network

\section{The lipopeptides strategy}

Since 1994 the French National Agency for AIDS research (ANRS's) vaccine strategy has aimed at inducing a multiepitopic cellular response by the administration of lipopeptides. Lipopeptides are hybrid molecules containing large synthetic fragments of viral proteins associated with a monopalmitoylated lipid chain, the two moieties being covalently bound. These synthetic constructs enhance immunogenicity without the use of an adjuvant. The lipid moiety enables a better penetration of peptides into the antigen-presenting cells (dendritic cells), thereby promoting the cellular immune response.

The ANRS vaccine strategy has been built over the years by relying on the results of multidisciplinary research combining the efforts of immunologists, biologists, and chemists.

In a series of original articles, research teams participating in an ANRS-supported network have described the use of binding/competition of peptides with HLA molecules to identify a series of polyepitopic regions of several human immunodeficiency syndrome (HIV) proteins (Choppin et al. 1991a, b). Immunogenicity of these peptidic fragments was assessed by measuring the induction of cytotoxic T lymphocytes (CTL) responses. In designing a vaccine strategy based on immunogenic pep-

${ }^{+}$Corresponding author. E-mail: elizabeth.fischer@anrs.fr Received 7 July 2004

Accepted 12 November 2004 tides inducing a polyclonal CTL response, our studies lead to the design of large peptide fragments containing "clusters" of epitopes corresponding to several HLA haplotypes. To avoid the risk of failure, it is necessary to select immunogenic epitopes in the most conserved regions of HIV. The peptide selection criteria include a strong affinity for class I molecules, the capacity to form a stable complex with these molecules, and the ability to induce CTL cytolytic activity.

Similar work was then performed to map in HIV proteins the CD4 epitopes that bind to class II MHC molecules. Regions containing the "clusters" of CD8 and CD4 epitopes for different haplotypes were identified and selected. These constructs were synthesized or inserted into different vectors to test their immunogenic potential in vivo.

The utilization of peptides in vaccine preparations is an alternative to recombinant vectors. The major advantages of a peptide vaccine are the specificity of the induced response and the low risk as compared to vaccines containing inactivated or attenuated viruses. Another advantage of this approach is that it allows the immune response to be directed against subdominant $\mathrm{T}$-cell epitopes. Lipopeptides are peptides linked by their carboxy-terminal to the lipid moiety comprising a palmitoyl group. A first in vivo study in mice demonstrated the immunogenic potential of lipopeptides (Martinon et al. 1992). Whereas the free peptides were weakly immunogenic, lipopeptides induced strong primary CTL responses allowing the bypass of the use of adjuvants. Lipopeptides of 16 or 34 amino acids were also effective for inducing immune responses, suggesting that several epitopes might be included in a single construct. T-helper responses (ac- 
tivation of CD4 lymphocytes) and B-cell responses (production of antibodies) were also achieved.

After the preliminary identification of regions rich in class I and class II HLA epitopes in HIV proteins, several lipopeptides were synthesized, each containing some thirty amino acids corresponding to immunogenic "agregotopes" derived from HIV proteins. Once mixed, the lipopeptides form micelles, which increases their solubility. These mixtures firstly evaluated in mice, induce a CD8 $\mathrm{T}$ cell response. Similarly, seven lipopeptides were synthesized, derived from known immunogenic regions of the SIV Nef and Gag proteins, and injected in rhesus macaques. The animals presented specific and functional CTL responses detectable up to 10 months after the injection (Bourgault et al. 1994). Antibodies were also observed in all immunized animals. In another experiment, the lipopeptides were optimized by addition of a T-helper peptide of tetanus toxoid (TT), and induced a strong CD4 response that was correlated with the induction of a multiepitopic CD8 response (Mortara et al. 1999). After infectious challenge, the responding macaques controlled the viral load better than the others. The lipopeptides are thus able to induce B- and T-cell responses in the primate.

Several patents have been derived from this research program belonging to French public research institutions which have licensed them to companies.

\section{Vaccine trials in humans}

The vaccine strategy based on lipopeptides has been evaluated in several trials sponsored by the ANRS. In most of these trials lipopeptides were combined with a viral vector in which are inserted genes that code for the same sequences as those that one finds in the lipopeptides. Hitherto, ANRS trials have used the canarypox vector
(Alvac) which was developed by Aventis-Pasteur with ANRS support. In the trials to come, the ANRS will use other viral vectors, such as modified vaccinia Ankara virus (MVA) or adenovirus in the same type of strategy of combining a viral vector and a lipopeptide.

Completed ANRS vaccine trials are shown in Table I.

ANRS VAC04 was a phase I trial in 28 healthy volunteers who received 3 injections of $6 \mathrm{Nef}, \mathrm{Gag}$, and Env lipopeptides (LIPO-6) (Gahery-Segard et al. 2000, Pialoux et al. 2001), and following data were observed: 1) an antibody response in $90 \%$ of the subjects; 2) anti-HIV CD4 response in $80 \%$ of the subjects; it was multispecific; 3 ) cytotoxic anti-HIV CD8 response tested by ELISPOT, and by means of conventional cytotoxicity tests, which was reproducible and multispecific in over half of the vaccines; 4) cytotoxic responses against Nef proteins derived from viruses other than the vaccine isolate were recorded in 3 of the 4 tested subjects tested, indicating the occurrance of immunization against conserved epitopes and suggesting a possible protection of the populations exposed to different HIV variants.

The lipopeptides therefore induce strong, lasting (over 2 years), multiepitopic and reproducible T-cell responses in a significant number of vaccinated subjects. The lipopeptides are well tolerated: the local and systemic reactions were weak and short-lived in these trials and similar to those induced by other candidate vaccines studied worldwide.

ANRS VAC09 was a study on memory response in which lipopeptides were used in booster doses in the volunteers of trials VAC03 and VAC07 trials who responded to the first vaccination comprising 3 to 5 injections of Alvac-HIV (vCP205 or vCP300). The results of this trial

TABLE I

Completed French National Agency for AIDS Research (ANRS) vaccine trials

\begin{tabular}{|c|c|c|}
\hline Trial & Immunogens & Aim \\
\hline ANRS VAC01 ${ }^{a}$ & $\operatorname{ALVAC}\left(\mathrm{vCP} 125^{c}\right)+\operatorname{rgp} 160$ & Prime-boost immunization limited to the envelope \\
\hline ANRS VAC02 ${ }^{a}$ & rgp $160+$ peptide V3 & $\begin{array}{l}\text { Prime-boost immunization limited to the envelope analogous to the } \\
\text { Protective envelope in chimpanzee }\end{array}$ \\
\hline ANRS VAC0 ${ }^{a}$ & $\begin{array}{l}\text { ALVAC }\left(\mathrm{vCP} 205^{d}\right) \\
+ \text { peptide CTLB-36 }\end{array}$ & Prime-boost, 1st attempt at multiple immunization \\
\hline ANRS VAC0 ${ }^{b}$ & 6 lipopeptides (Env, Gag, Nef) & 1st attempt at multiple immunization using a mixture of lipopeptides \\
\hline ANRS VAC0 $5^{a}$ & $\begin{array}{l}\text { ALVAC (vCP125) or control } \\
\text { ALVAC (vCP rage) }\end{array}$ & Study on memory response in the vaccinated subjects from trial VAC01 \\
\hline ANRS VAC06 ${ }^{a}$ & $\begin{array}{l}\text { ALVAC (vCP125) or control } \\
\text { ALVAC (vCP rage) }\end{array}$ & Study on memory response in the vaccinated subjects from trial VAC02 \\
\hline ANRS VAC07 ${ }^{a}$ & $\operatorname{ALVAC}\left(\mathrm{vCP} 300^{f}\right)$ & 1st attempt at multiple immunization containing CTL domains \\
\hline ANRS VAC08 ${ }^{a}$ & $\begin{array}{l}\text { ALVAC (vCP205) or peptide } \\
\text { CTLB-36 }\end{array}$ & Study on memory response in the vaccinated subjects from trial VAC03 \\
\hline ANRS VAC0 ${ }^{b}$ & 6 lipopeptides (Env, Gag, Nef) & $\begin{array}{l}\text { Study on memory response in the vaccinated subjects from trials } \\
\text { VAC03 and VAC07 }\end{array}$ \\
\hline
\end{tabular}


have shown that lipopeptides can induce a booster effect. This is an advantage compared with the recombinant vaccines Alvac and adenovirus, which need increasingly high vaccine doses to overcome the obstacle of immunization against the vector.

In view of these promising results, ANRS has determined to pursue the phase I and II vaccine trials in healthy volunteers using lipopeptides. At least eight phase I or II trials are under way, or they should begin this year (Table II).

These trials concern the formulations, doses and routes of administration of the lipopeptides, alone or combined with the vector Alvac in a prime-boost strategy.

ANRS trials VAC10 and VAC12 evaluated the toxicity and immunogenicity of new preparations of lipopeptides comprising different HIV-1 peptides. The aim was to induce potent $\mathrm{CD} 4$ and $\mathrm{CD} 8$ responses. As the in vivo induction of CTL (CD8+) necessitates the concomitant stimulation of a CD4 T-helper response, a CD4+ epitope of the tetanus toxin (TT) was added. For VAC10, a TT lipopeptide was added to the LIPO-5 vaccine preparation, while for VAC12 the TT peptide was integrated collinearly to each lipopeptide of interest. These trials have already finished, and data analysis is under way.

ANRS trial VAC17 tested the memory response in vaccinated subjects from trial VAC10. Data analysis is under way.

ANRS trial VAC16 compares the intradermal and intramuscular injection routes to determine which one will be the most effective for immunization and/or uses the least vaccine.

ANRS trial VAC18 is a phase II trial for determining the minimum lipopeptide dose that produces optimal immunization (dose-ranging study with LIPO-5).

Trial ANRS VAC19/HVTN 042 is a phase II trial designed to confirm the results of trial VAC10 concerning candidate vaccine LIPO-5 in a double-blind design and in a large number of American volunteers. This lipopeptide has also tested in a prime-boost vaccine strategy with a dose-ranging study of the boost, being Alvac vCP1452 used for the primary immunization

In addition, two trials exploring mucosal immunity are ongoing in 2004: ANRS trial Pre-VAC14 is evaluating the feasibility of mucosal sampling techniques; ANRS trial VAC14 is designed to study the mucosa as a new HIV-1 immunization route and the immune responses induced at the mucosal sites. The vaccine preparation has been administered to female HIV-seronegative volunteers by the nasal or vaginal mucosal route, and mucosal immunity has been evaluated in samples collected from nose, saliva and vagina.

ANRS will pursue the phase I/II trials using a primeboost strategy combining lipopeptides with viral vectors (MVA, adenovirus). The teams supported by ANRS have recently tested the original hypotheses concerning the utilization of lipopeptides combined with adenoviruses. The latter potentiate the immune response induced by with lipopeptides (Molinier-Frenkel et al. 2002).
ANRS has also been considering the utilization of adjuvants for cellular immunity so as to enhance the efficacy of the lipopeptides. This strategy means that new industrial partnerships can be envisaged. By the year 2005, the priority will be an MVA/lipopeptides combination taking advantage of the fact that there are MVAs approved for human clinical trials. The phase I/II trials will be continued in partnership with one of the large vaccine companies, until a decision will be taken to move towards an efficacy trial by the year 2008 .

\section{The ANRS vaccine research network}

ANRS has set up a genuine network linking all the people and institutions concerned on basic to clinical research. This network enables the coordination of all scientific projects, and the organization of research on complementary topics (chemistry, immunology, animal experimentation). It also enables the involved research teams to share their own resources, and to be benefited from common technological platforms that are constantly updated with new techniques developed in the laboratories. The network promotes innovation and adaptation of programs through the sharing and common utilization of data and observations. The Preventive Vaccine Network has been organized on six main themes:

The AC28 "Preventive vaccine trials" - This scientific committee is in charge of evaluating and approving preventive vaccine trial proposals. Since 2001, the ANRS has sponsored all the vaccine trials set up within the framework of its vaccine program.

Preclinical research - The Preventive Vaccine Network links laboratories involved in four main fields of research: the design of lipopeptides, and the study on the mechanisms underlying their immunogenicity; the design of recombinant vectors; the development of new techniques to evaluate the response in vaccinated subjects ; the evaluation of the impact of viral variability on T-cell recognition.

Clinical centers - Six clinical centers are involved in the vaccine research of the ANRS. They recruit volunteers and participate in the vaccine trials. There are three centers in Paris and three elsewhere (Nantes, Marseille, and Toulouse).

The network of volunteers - The setting up of vaccine trials is contingent upon recruitment of suitable volunteers. In 1992 the ANRS set up a multidisciplinary committee to select candidates. ANRS representatives, clinicians, and psychiatrists/psychologists sit on this committee. The volunteers in whom the candidate vaccines are evaluated must be HIV-seronegative and not exposed to risks of contamination, and must understand and accept the risks associated with the research. The volunteer selection procedure takes into account the four main risks: biological risk, the vaccine preparations tested may induce the production of antibodies which, in the case of contact with HIV, might facilitate contamination; behavioral risk, a volunteer who believes him/herself to be unduly protected by a candidate vaccine could adopt behaviors that heighten the risk of HIV contamination; psy- 
TABLE II

Ongoing French National Agency for AIDS Research (ANRS) vaccine trials

ANRS VAC10

(phase I, sponsor: Aventis-Pasteur, trial ended in January 2003)

Aim

Number of volunteers

Methodology

Candidate vaccine

Immunization regimen

To test a new mixture of lipopeptides to which can be added a lipopeptide bearing a T-helper epitope 60 volunteers (divided into 4 groups)

Single-center, comparative, randomized, open trial

Recombinant canarypox ALVAC (vCP1452) expressing the envelope protein gp120 associated with the transmembrane part of gp41, Gag protein, CTL domains of Nef and Pol

LIPO-5: mixture of 5 synthetic lipopeptides whose peptide sequences represent CTL epitopes contained in HIV-1 proteins Gag (aa 17-35 + aa 253-284), Pol (aa 325-355) and Nef (aa 66-97 + 116-145). At the C-terminal each peptide is linked to monopalmitic acid via a lysine

LIPO-6T: LIPO-5 completed by a lipopeptide corresponding to a CD4 epitope of tetanus toxin (aa 830-846).

5 injections at M0, M1, M3, M6 and M12 with vCP1452 or LIPO-5 or LIPO-6T or LIPO-6T + vCP1452 and follow-up till M24

\section{ANRS VAC12}

(phase I, sponsor: Biovector Therapeutics, trial ended in August 2002)

\section{Aim}

To test a new mixture where each lipopeptide is associated with a T-helper epitope

Number of volunteers

15

Methodology

Single-center, open trial

Candidate vaccine

LPHIV1: mixture of 4 synthetic lipopeptides whose peptide sequences are contained in HIV-1 proteins Gag (aa 77-85), Pol-RT (aa 342-354), Pol (aa 476-484) and Nef (aa 62-82). Each peptide is collinearly associated with a T-helper epitope of tetanus toxin (aa 830-843) which is linked to monopalmitic acid at the N-terminal.

Immunization regimen

3 injections at M0, M1 M3 and follow-up till M12

ANRS VAC17

(phase I, sponsor: ANRS, trial ended in October 2002)

\begin{tabular}{ll} 
Aim & To test the memory response to lipopeptides in vaccinated subjects from trial VAC10 \\
Number of volunteers & $\begin{array}{l}35: 25 \text { from } 4 \text { groups of VAC10 and } 10 \text { naive for LIPO-6T antigens (control group) } \\
\text { Methodology }\end{array}$ \\
Single-center, comparative, open trial \\
Immunization regimen & LIPO-6T: see VAC10 \\
\hline
\end{tabular}

ANRS VAC19/HVTN042

(phase II, sponsor NIAID (USA), trial started in March 2004)

\section{Aim}

Number of volunteers

Methodology

Candidate vaccine

Immunization regimen
To confirm on a larger scale the results previously recorded with LIPO-5, by means of a prime-boost vaccine strategy with a dose-ranging study of the boost

180 (divided into 10 groups : $5 \times 30$ immunized volunteers $+5 \times 6$ placebo volunteers)

Multicenter, comparative, randomized, double-blind trial versus placebo

Recombinant canarypox ALVAC (vCP1452): see VAC10 LIPO-5: see VAC10Placebo

Single immunization with $500 \mu \mathrm{g}$ of LIPO- 5 or 1 dose of ALVAC at W0, W4, W12 and W24 or primeboost immunization with 1 dose of ALVAC at W0 and W4 then booster doses of 500, 150 or $50 \mu \mathrm{g}$ of LIPO-5 at W12 and W24. Follow-up till M18
Aim

Number of volunteers

Methodology (phase IB, sponsor ANRS, trial started in July 2004)

\section{ANRS VAC16}

To determine which of the intradermal and intramuscular injection routes will be the most effective in immunization and/or using the least vaccine

70 (divided into 2 groups)

Multicenter, randomized, stratified, open trial 


\begin{tabular}{|c|c|}
\hline Candidate vaccine & LPHIV1: see VAC12 \\
\hline Immunization regimen & 3 injections at W0, W4 and W12 by IM or ID route and follow-up till W24. \\
\hline & $\begin{array}{c}\text { ANRS VAC18 } \\
\text { (phase II, sponsor ANRS, trial started in September 2004) }\end{array}$ \\
\hline Aim & To determine the optimal minimum dose (dose-ranging study) \\
\hline Number of volunteers & 132 (divided into 4 groups) \\
\hline Methodology & Multicenter, comparative, randomized, double-blind trial versus placebo \\
\hline Candidate vaccine & LIPO-5: see VAC10Placebo: diluent of LIPO-5, i.e. $5 \%$ glucose solution buffered in $20 \mathrm{mM}$ Tris/HCl \\
\hline Immunization regimen & $\begin{array}{l}4 \text { injections at W0, W4, W12 and W24 with } 500 \text { or } 150 \text { or } 50 \text { or } 0 \mu \mathrm{g} \text { of LIPO-5 and follow-up till } \\
\text { W48 }\end{array}$ \\
\hline
\end{tabular}

chological pressure, participants in vaccine trials may produce anti-HIV antibodies. They are in a situation of "pseudo-seropositivity" or "seropositivity without the virus". A screening test may be positive even though they are not carrying the virus; social pressure, the situation of pseudo-seropositivity may prove problematical in certain situations (crossing the borders of certain countries, for instance, or obtaining insurance when applying for a loan).

The selection is done based on a written form, in which the volunteers explain their reasons for wanting to participate in the trials, and then on the basis of laboratory test findings and the interviews with a clinician and a psychiatrist or psychologist. The volunteers are not paid. So far, just over 600 people have joined the network of volunteers.

Platforms for evaluation of immune responses - The immune response in volunteers vaccinated during clinical trials has been assessed at three sites, using standardized protocols: (i) the "cellular immunology" platform has been evaluating the CD4 and CD8 responses in immunized volunteers by various methods: T-cell proliferation, cytolysis tests (CTL), Elispot CD8+ and CD4+ and intracellular labeling of cytokines; (ii) the "antibody " platform has been exploring the specificity of the produced antibodies, and the neutralizing activity from sera of vaccinated subjects; (iii) the "mucosal immunology" platform has been investigating the humoral mucosal immunity by means of techniques, such as: the quantification of $\operatorname{IgA}$ and IgG, analysis of their specificity and isotype profiles, functional assays of inhibition of viral transcytosis, and of inhibition of virion transfer from dendritic cells to $\mathrm{T}$ lymphocytes.

Research in the developing world - Research in the developing countries is one of the priorities of the ANRS, which therefore intends to incorporate in its vaccine program those countries in which there are ANRS sites. Clinical trials in these countries, notably Ivory Coast, Senegal, and Cameroon, are planned. This initiative is part of the planned realization of phase III trials designed to evaluate the efficacy of candidate vaccines. The vaccine program in the developing countries is mainly centered on the Abidjan site and currently involving: (i) the analysis of the viral sequences present in the Ivory Coast population. The first data indicate that the sequences of Gag and Nef seem to be well conserved between the AG strains, predominant in Ivory Coast, and the B strains, predominant in France; (ii) the evaluation of the immune response to determine whether the utilization of lipopeptides designed from sequences of the subtype B virus is relevant in populations infected by other viral subtypes; (iii) setting up an infrastructure for the recruitment of volunteers and for a future clinical trial.

\section{The industrial partners}

The vaccine research program has been founded on collaborations with industrial partners. Over more than ten years, successive agreements have been drawn up between the ANRS and Aventis-Pasteur in order to set up the clinical development programs necessary to progress in the use of lipopeptides. ANRS has also financed certain aspects of the fundamental research pursued by Aventis-Pasteur. This collaboration has particularly enabled Aventis-Pasteur to develop the recombinant vectors (Alvac) currently used in several international phase II and III trials.

At the same time, partnerships have been forged with other companies, such as Biovector Therapeutics. This has accelerated the clinical development of lipopeptides by diversifying access to clinical batches. Presently, transgene company has been developing clinical lots of MVA containing the same viral sequences as those inserted into canarypox.

\section{International collaborations}

A number of international collaborations have been established with: 1) The American HIV Vaccine Trial Network (HVTN). The collaboration relates notably to the standardization of immunological techniques used for evaluating cellular immunity, and for exchanging of protocols and reagents. In the HVTN042/ANRS VAC19 trial, the evaluation of the immune response will be done in tandem in the United States and in France; 2) Canvac, the Canadian network for the production of vaccines and immunotherapies. The collaboration concerns the mucosal immunity, and it involves standardization of the different methods of mucosal sampling, definition of the 
practicalities of the functional study on CTL from peripheral mucosa, and the comparison and the implementation of methodological approaches to explore the function of antibodies in mucosal sites.

\section{REFERENCES}

Bourgault I, Chirat F, Tartar A, Levy JP, Guillet JG, Venet A 1994. Simian immunodeficiency virus as a model for vaccination against HIV. Induction in rhesus macaques of GAGor NEF-specific cytotoxic T lymphocytes by lipopeptides. J Immunol 152: 2530-2537.

Choppin J, Martinon F, Connan F, Gomard E, Levy JP 1991a. HLA-binding regions of HIV-1 proteins. I. Detection of seven HLA binding regions in the HIV-1 Nef protein. $J$ Immunol 147: 569-574.

Choppin J, Martinon F, Connan F, Pauchard M, Gomard E, Levy JP 1991b. HLA-binding regions of HIV-1 proteins. II. A systematic study of viral proteins. J Immunol 147: 575583.

Gahery-Segard H, Pialoux G, Charmeteau B, Sermet S, Poncelet H, Raux M, Tartar A, Levy JP, Gras-Masse H, Guillet JG 2000. Multiepitopic B- and T-cell responses induced in humans by a human immunodeficiency virus type 1 lipopeptide vaccine. J Virol 74: 1694-1703.

Martinon F, Gras-Masse H, Boutillon C, Chirat F, Deprez B, Guillet JG, Gomard E, Tartar A, Levy JP 1992. Immunization of mice with lipopeptides bypasses the prerequisite for adjuvant. Immune response of BALB/c mice to human immunodeficiency virus envelope glycoprotein. J Immunol 149: 3416-3422.

Molinier-Frenkel V, Lengagne R, Gaden F, Hong SS, Choppin J, Gahery-Segard H, Boulanger P, Guillet JG 2002. Adenovirus hexon protein is a potent adjuvant for activation of a cellular immune response. J Virol 76: 127-135.

Mortara L, Gras-Masse H, Rommens C, Venet A, Guillet JG, Bourgault-Villada I 1999. Type 1 CD4(+) T-cell help is required for induction of antipeptide multispecific cytotoxic $\mathrm{T}$ lymphocytes by a lipopeptidic vaccine in rhesus macaques. J Virol 73: 4447-4451.

Pialoux G, Gahery-Segard H, Sermet S, Poncelet H, Fournier S, Gerard L, Tartar A, Gras-Masse H, Levy JP, Guillet JG; ANRS VAC 04 Study Team 2001. Lipopeptides induce cell-mediated anti-HIV immune responses in seronegative volunteers. Aids 15: 1239-1249. 